\title{
Overexpression of $e$ IF3D in Lung Adenocarcinoma Is a New Independent Prognostic Marker of Poor Survival
}

\author{
Dan Wang $\mathbb{D}^{1}{ }^{1}$ Yichen Jia, ${ }^{2}$ Wendan Zheng, ${ }^{3}$ Changzheng Li, ${ }^{1}$ and Wen Cui ${ }^{1}$ \\ ${ }^{1}$ Institute of Forensic Medicine and Laboratory Medicine, Jining Medical University, Hehua Road 133\#, Jining City, \\ 272067 SD, China \\ ${ }^{2}$ Institute of Medical Technology, Qiqihar Medical University, Jianhua District, Qiqihar City, 161006 HLJ, China \\ ${ }^{3}$ The Second Clinical Medical College, Jining Medical University, Hehua Road 133\#, Jining City, 272067 SD, China \\ Correspondence should be addressed to Wen Cui; cuiwenmdd@163.com
}

Received 19 June 2019; Revised 2 September 2019; Accepted 26 September 2019; Published 7 December 2019

Academic Editor: Yi-Chia Huang

Copyright (c) 2019 Dan Wang et al. This is an open access article distributed under the Creative Commons Attribution License, which permits unrestricted use, distribution, and reproduction in any medium, provided the original work is properly cited.

\begin{abstract}
The eukaryotic initiation factor 3 (eIF3) is the largest and most complex translation initiation factor in mammalian cells. It consists of 13 subunits and among which several were implicated to have significant prognostic effects on multiple human cancer entities. To examine the expression profiles of eIF3 subunits and determine their prognostic value in patients with lung adenocarcinoma (LUAD), the genomic data, survival data, and related clinical information were obtained from The Cancer Genome Atlas (TCGA) project for a secondary analysis. The results showed that among ten aberrantly expressed eIF3 subunits in tumours compared with adjacent normal counterparts $(p<0.05)$, only upregulated eIF3D could predict poor overall survival (OS) outcome independent of multiple clinicopathological parameters $(\mathrm{HR}=2.043,95 \% \mathrm{CI}: 1.132-3.689, p=0.018)$. Chi-square analysis revealed that the highly expressed $e I F 3 D$ group had larger ratios of patients with advanced pathological stage $(68 / 40$ vs. 184/206, $p=0.0046)$, residual tumour $(13 / 4$ vs. $163 / 176, p=0.0257)$, and targeted molecular therapy $(85 / 65$ vs. $138 / 164, p=0.0357)$. In silico analysis demonstrated that the altered expression of eIF3D was at least regulated by both copy number alterations (CNAs) and the hypomethylation of cg14297023 site. In conclusion, high eIF3D expression might serve as a valuable independent prognostic indicator of shorter OS in patients with LUAD.
\end{abstract}

\section{Introduction}

Lung cancer is one of the most common and lethal diseases afflicting the global population. It is histologically divided into small cell lung cancer (SCLC) and non-small-cell lung cancer (NSCLC). Lung adenocarcinoma (LUAD) is the most common subtype of NSCLC, accounting for more than $40 \%$ of NSCLC cases and showing a clear upward trend in the past few decades [1]. The overall survival (OS) rate for LUAD patients remains low despite the advances in clinical treatment. Due to the insignificant early symptoms and the lack of specific markers, the early diagnosis of LUAD is extremely difficult, and patients usually have a stage of local progression or later when they are diagnosed, missing the best time of surgery [2-4]. Therefore, the search for new and effective biomarkers to screen out high-risk patients and predict prog- nosis of LUAD has become an important part of lung cancer prevention and treatment.

mRNA translation is a key step in the regulation of eukaryotic gene expression. Its dysregulation would result in abnormal gene expression and lead to uncontrolled cell growth, which potentially caused tumourigenesis [5]. The initiation of mRNA translation is the rate-limiting step during which translation regulation is primarily achieved and its dysregulation has received considerable attention, as well as aberrant expression of eukaryotic translation initiation factors (eIFs) in various cancer entities has been observed in a growing number of studies [6-14]. eIF3, the largest and most complex eIF in mammalian cells, consists of 13 nonidentical subunits (eIF3a-m). The main function of eIF3 has been demonstrated to promote nearly every step of translation initiation as a scaffold, including promoting the $43 \mathrm{~S}$ 
preinitiation complex formation, stimulating mRNA binding with $43 \mathrm{~S}$ preinitiation complex, scanning and recognizing AUG start codon, and dissociating the posttermination $80 \mathrm{~S}$ ribosome by binding with $40 \mathrm{~S}$ ribosomal subunit [15-18]. The altered expression of eIF3 subunits has been found in a wide range of tumour entities, including breast cancer [19-21], gastric cancer [22], NSCLC [23], and liver cancer [24], supporting their high potential use as prognostic biomarkers and therapeutic cancer targets. To the best of our knowledge, no previous studies have examined the roles of overall eIF3 family members in LUAD. In this study, using gene expression data and survival data of LUAD patients from The Cancer Genome Atlas (TCGA) project, we examined the expression profiles of eIF3 subunits and determined their prognostic roles in LUAD, as well as the underlying mechanisms of eIF3D dysregulation.

\section{Materials and Methods}

2.1. Clinical Cohorts and RNA-Seq Data from TCGA. mRNA expression data, survival data, and related clinical information of patients with LUAD in TCGA project were downloaded from the UCSC Xena browser (https://xenabrowser.net/). Only patients who had primary tumours and had not received neoadjuvant therapy were included in this analysis. The genomic and phenotypic data were collected. Briefly, RNA-Seq data of eIF3 subunit genes were extracted. The phenotypic data included sample types, histological types, age at initial pathologic diagnosis, gender, pathologic stage, radiation therapy, targeted molecular therapy, residual tumour status, canonical mutations in KRAS/EGFR/ALK, tobacco smoking history, OS status, and OS time (weeks). To explore the underlying mechanisms of eIF3D dysregulation, the corresponding somatic mutations, copy number alterations (CNAs), and DNA methylations of eIF3D were obtained. The DNA methylation status was examined by the Infinium Human Methylation 450 Bead Chip.

2.2. Statistical Analysis. Statistical analysis was performed using GraphPad Prism 8 (GraphPad Inc., CA, USA) and SPSS Statistics 20 (SPSS Inc., IL, USA) in this study. Comparison between two groups was performed using unpaired $t$-test with Welch's correction. The differences in clinicopathological parameters between LUAD patients with high or low gene expression were compared using the chi-square test by two-sided Fisher's exact test. Gene expression levels were categorized as low or high according to the median values. Kaplan-Meier curves of OS were generated using GraphPad Prism, and differences in survival rate between groups were analysed by log-rank test. Univariate and multivariate Cox regression models were utilized to assess the prognostic value of eIF3 subunits regarding OS. Genes with a $p$ value of $<0.05$ in log-rank test were subsequently included in univariate and multivariate Cox analyses. Forest plots for multivariate Cox regression models were also drawn by GraphPad Prism. Pearson correlation coefficients between eIF3D expression and its linear DNA copy number values in patients, as well as the methylation level of its CpG site cg14297023, were calculated to assess their correla- tions. $p<0.05$ indicates statistically significant for all above statistical analyses.

\section{Results}

3.1. The mRNA Expression Levels of eIF3 Subunits in LUAD Patients. Using RNA-Seq data in TCGA, the mRNA expression levels of all 13 eIF3 subunits were compared between LUAD $(n=511)$ and adjacent normal tissues $(n=58)$. As shown in Figure 1, levels of nine eIF3 subunits involving $e I F 3 A, e I F 3 B, e I F 3 C, e I F 3 D, e I F 3 E, e I F 3 H, e I F 3 I, e I F 3 J$, and eIF3 $M$ were significantly increased in cancer tissues, whereas the eIF3L expression level in tumours was independently decreased than that of normal tissues $(p<0.05)$. No significant variation was observed in $e I F 3 F, e I F 3 G$, and $e I F 3 K$ levels between the two groups $(p \geq 0.05)$.

3.2. Association between eIF3 Subunit Expression Levels and Clinical Features in LUAD Patients. Next, we summarized the association between gene expression levels of the 13 eIF3 subunits and clinicopathological parameters of LUAD patients (Table 1). Chi-square analysis showed that five subunit genes associated significantly with a single clinicopathological variable, respectively, and among which eIF3C and eIF3H expression increased in high pathological stage (eIF3C: $64 / 44$ vs. $188 / 207, p=0.0388$; eIF3H: $67 / 41$ vs. $186 / 209, p=0.0066), e I F 3 F$ and $e I F 3 L$ expression increased in patients with ages no lower than 65 (eIF3F: 144/129 vs. 94/125, $p=0.0367$; eIF3L: $151 / 122$ vs. 93/126, $p=0.0050$ ), while high expression of $e I F 3 G$ was observed more in female patients $(157 / 120$ vs. $102 / 132, p=0.0034)$.

Among the five genes whose expression levels were significantly associated with two clinicopathological variables or more, the high eIF3A expression group had larger proportion of male patients $(134 / 100$ vs. $120 / 157, p=0.0019)$ and patients with smoking history ( $220 / 202$ vs. $29 / 46, p=0.0336)$, while the expression of the other four genes was consistently positively associated with high pathological stage (eIF3B: 66/42 vs. $189 / 206, p=0.0168$; eIF3D: $68 / 40$ vs. $184 / 206, p=0.0046$; eIF3E: $64 / 44$ vs. $186 / 209, p=0.0297$; eIF3J: $63 / 45$ vs. $183 / 212, p=0.0299)$. Besides, the more highly expressed eIF3B was also discovered in patients with ages lower than $65(126 / 93$ vs. $122 / 151, p=0.0050)$, radiation therapy (37/21 vs. $194 / 202, p=0.0483)$, and targeted molecular therapy $(87 / 63$ vs. $143 / 159, p=0.0361)$. The high eIF3D expression group had a significantly larger ratio of patients with residual tumour $(13 / 4$ vs. $163 / 176, p=0.0257)$ and targeted molecular therapy $(85 / 65$ vs. $138 / 164, p=0.0357)$. And high expression of eIF3E and eIF3J was observed more in patients with residual tumours (13/4 vs. $172 / 170, p=0.0456)$ and without mutations in KRAS/EGFR/ALK (47/48 vs. 86/46, $p=0.0206)$, respectively. No significant association was observed between eIF3I, eIF3K, IIF3M, and other above-mentioned clinical features.

3.3. The Prognosis Value of eIF3 Subunit Expression in LUAD Patients. Since we observed that ten eIF3 subunits were significantly aberrantly expressed in tumours compared with adjacent area, we next evaluated the association between 


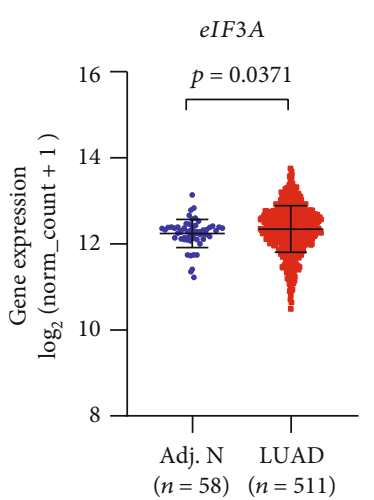

(a)

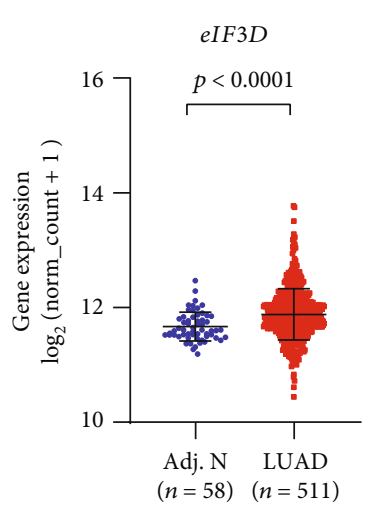

(d)

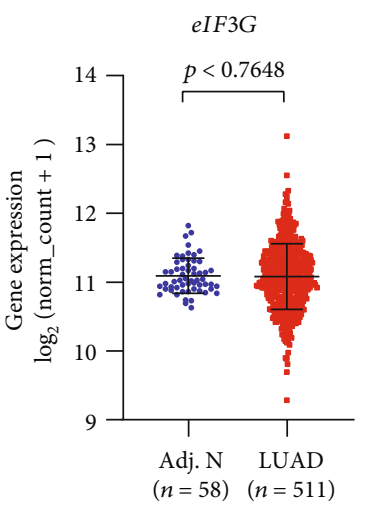

(g)

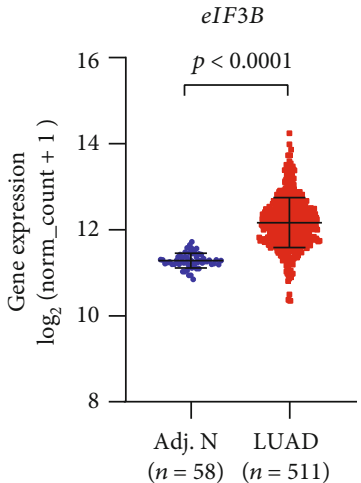

(b)

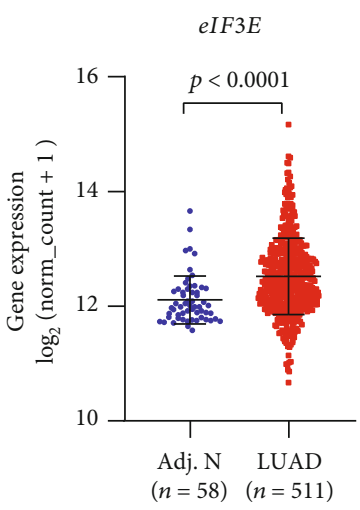

(e)

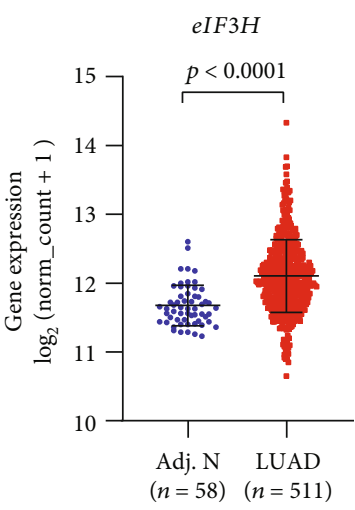

(h)

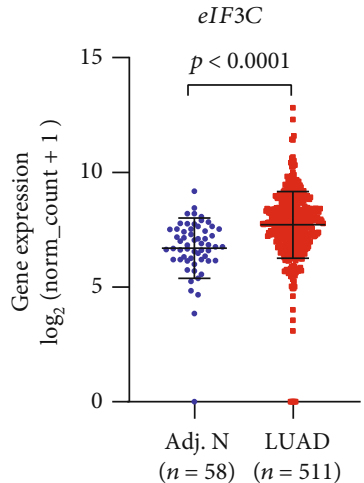

(c)

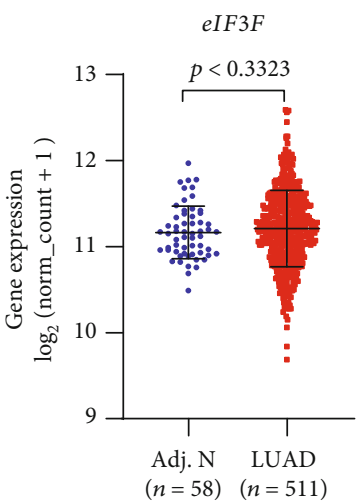

(f)

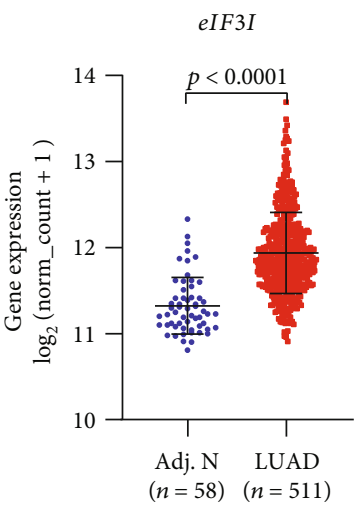

(i)

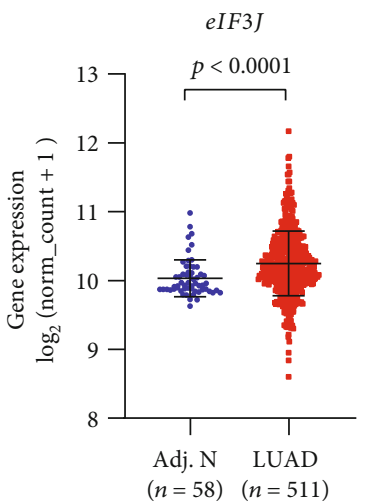

(j)

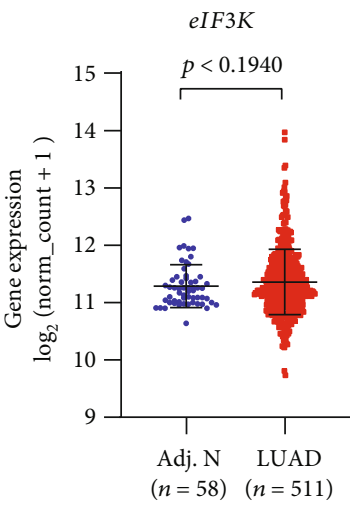

(k)

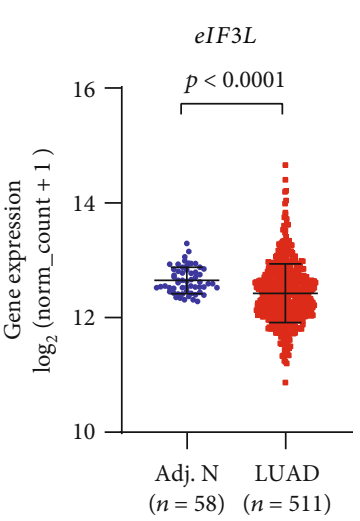

(1)

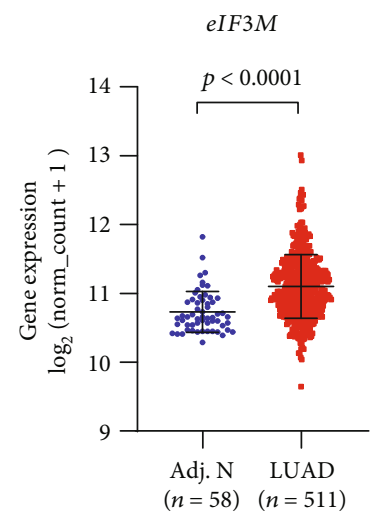

(m)

FIGURE 1: Plot charts showing mRNA expression of eIF3 subunits in LUAD tissues and adjacent normal tissues. Adj. N: adjacent normal tissues. 


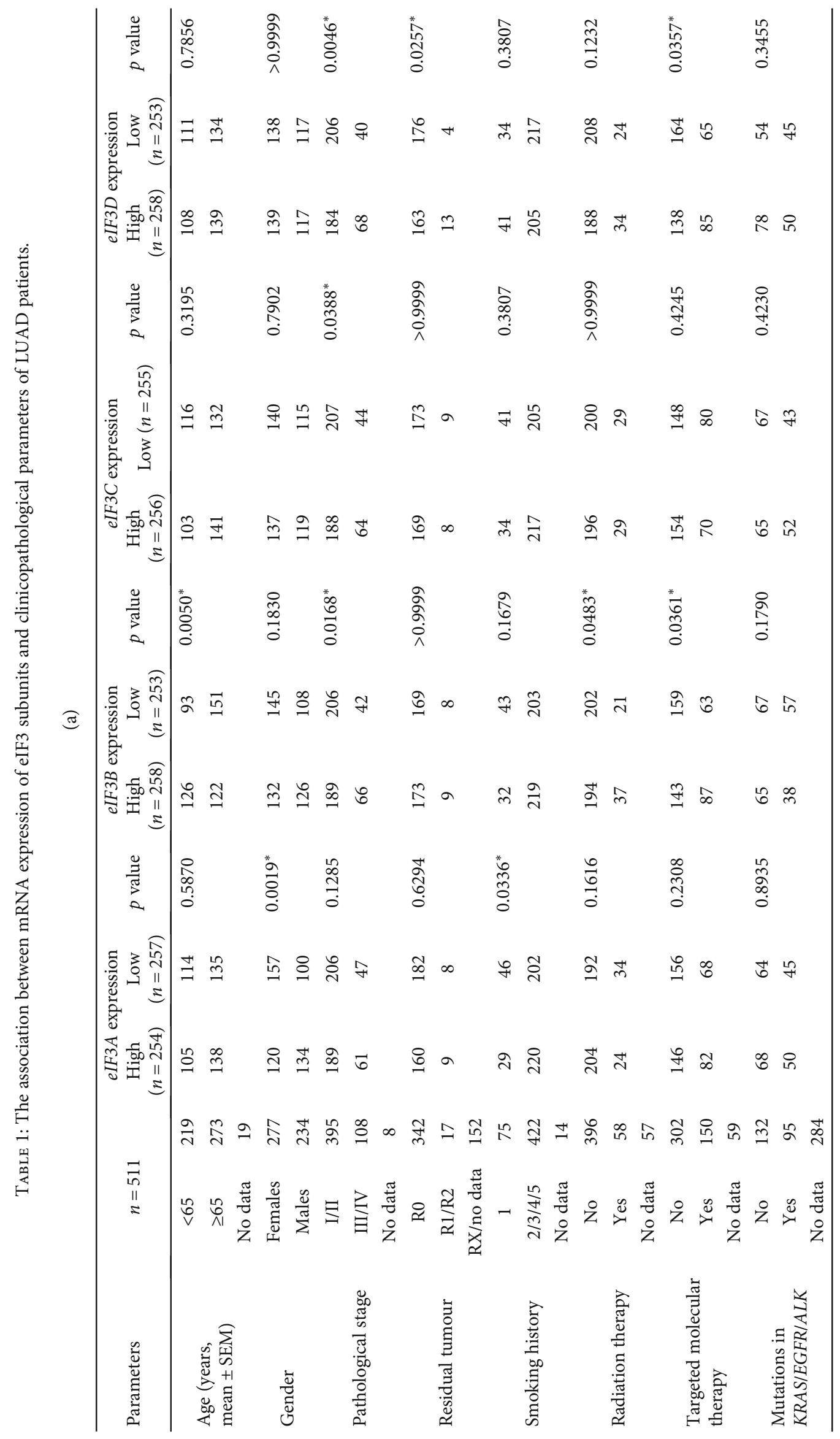




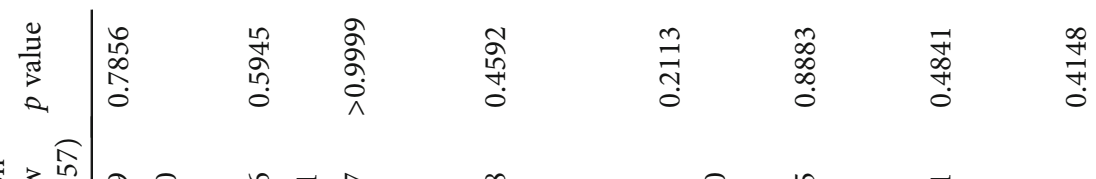

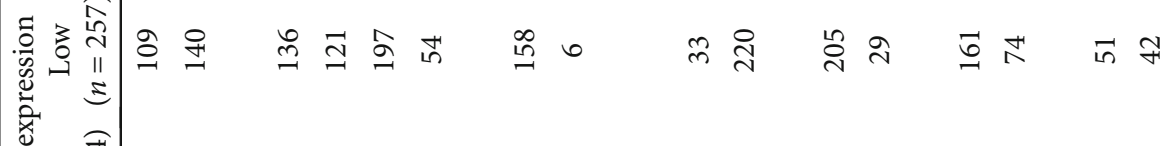

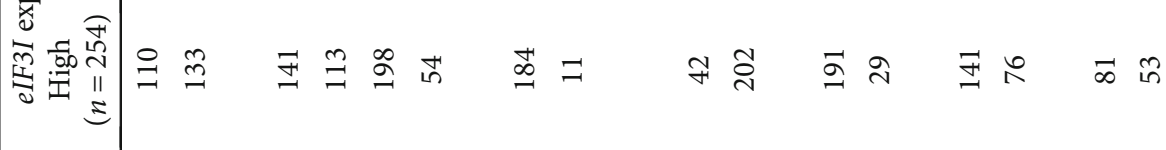

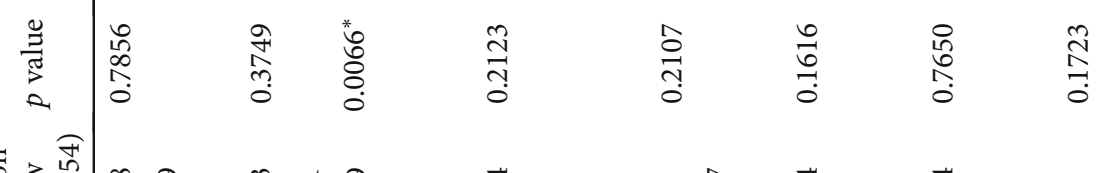

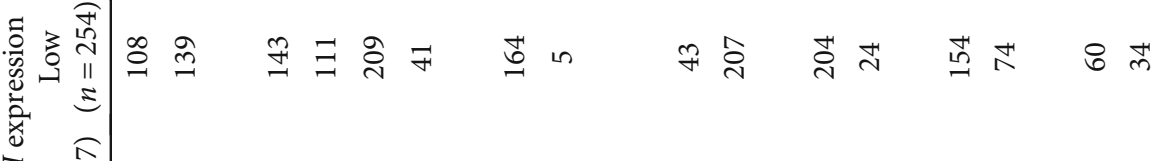

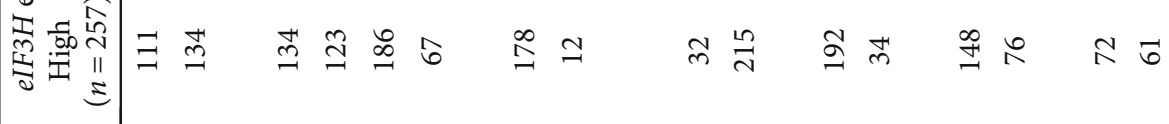

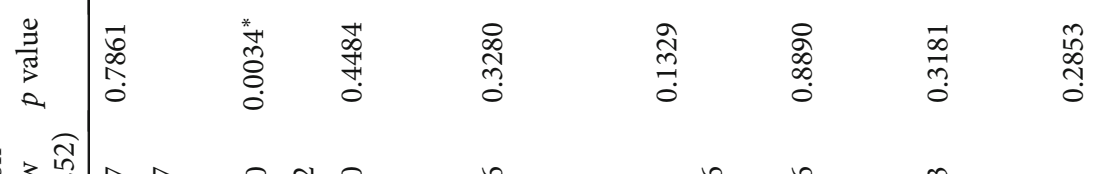

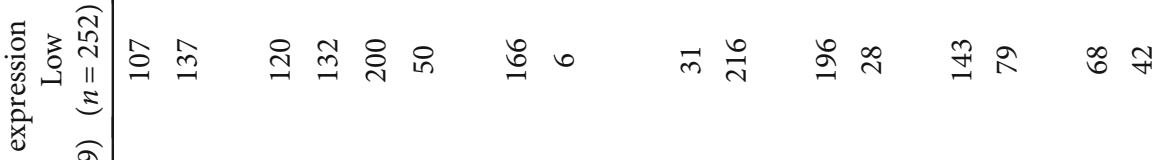

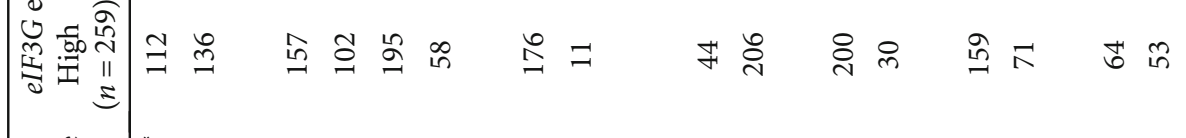

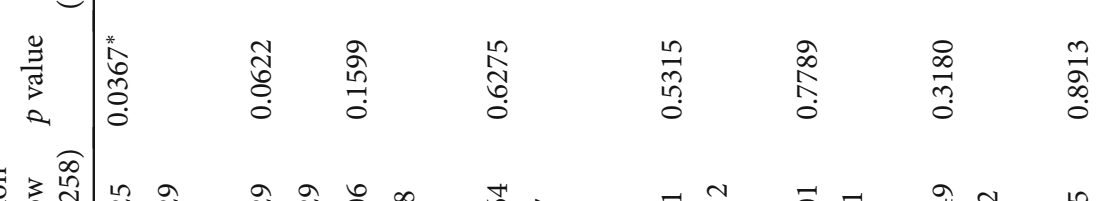

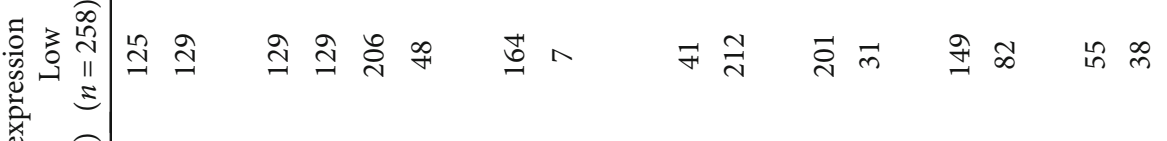

焉点点

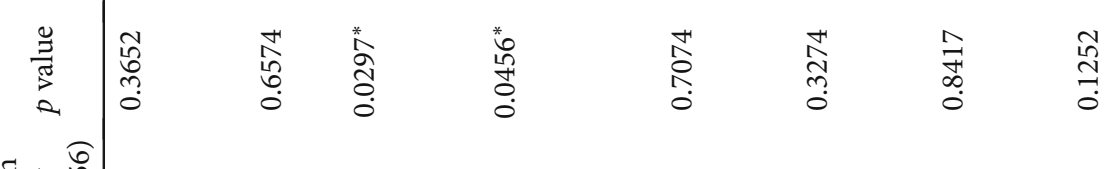

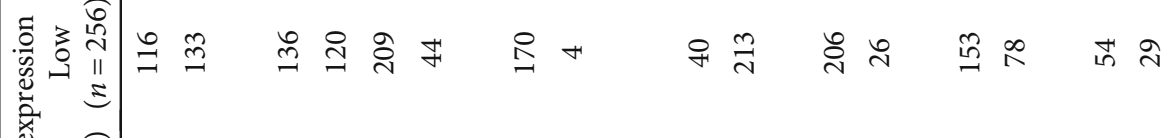

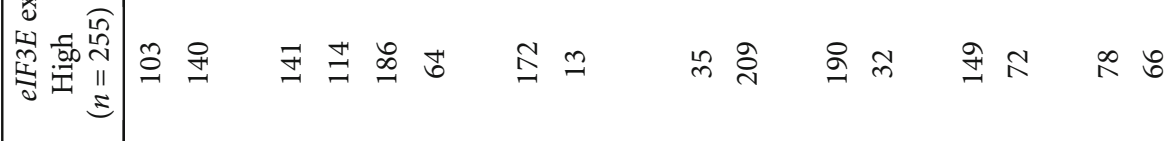

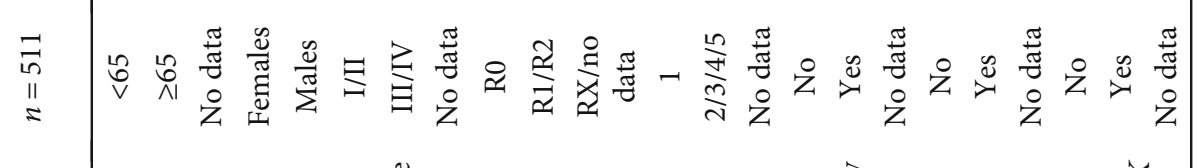

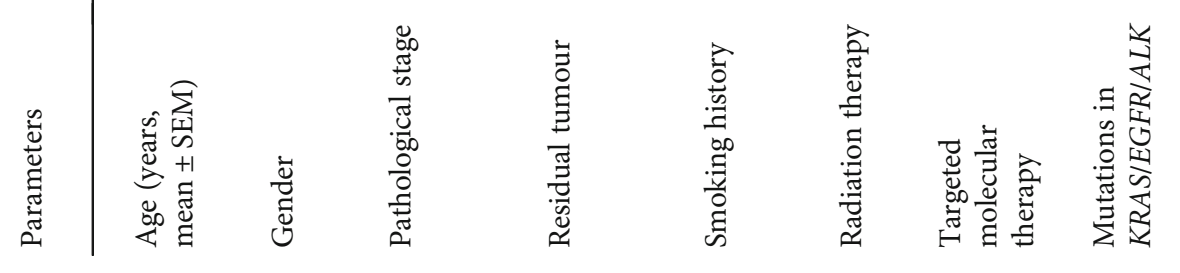




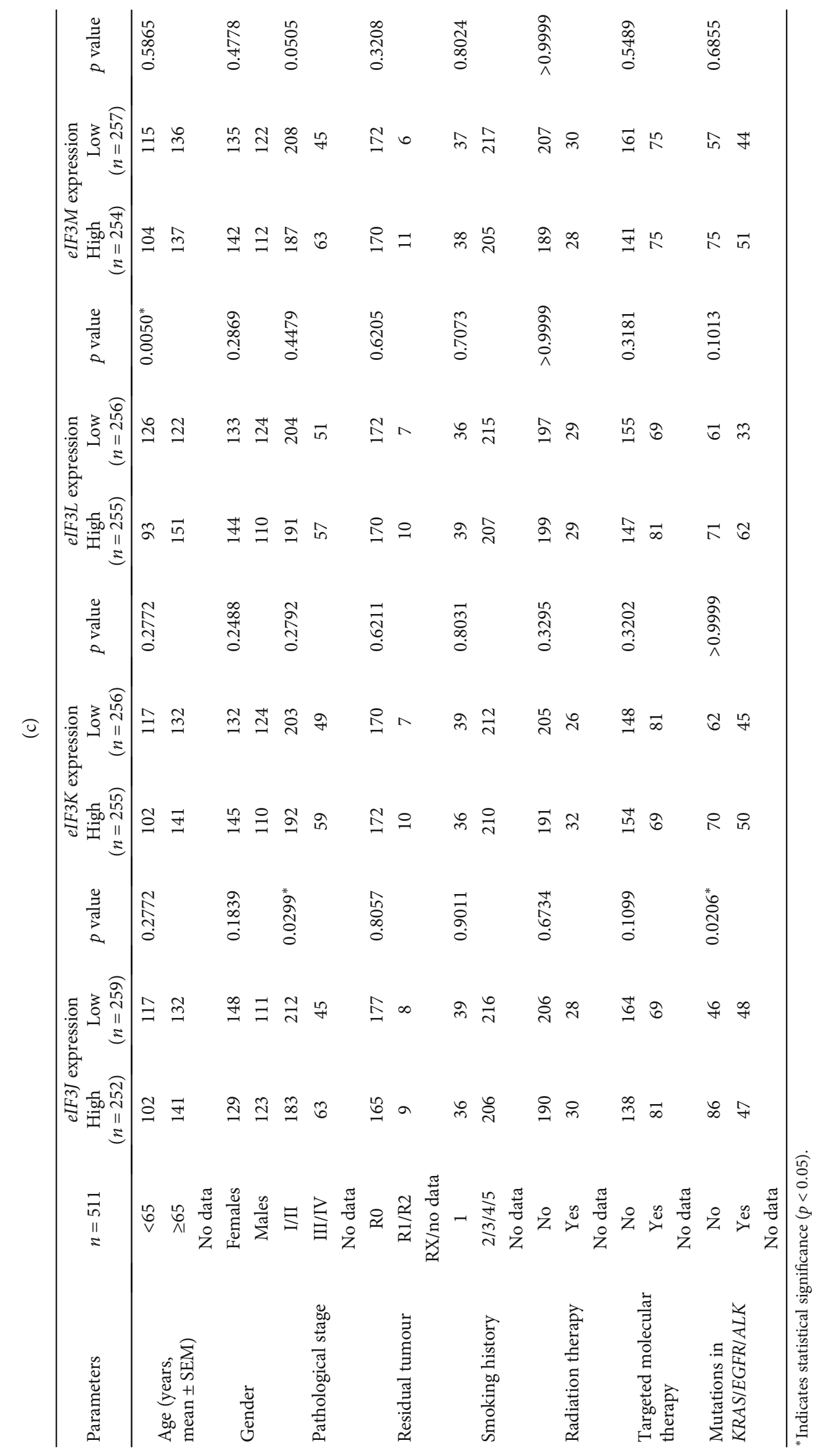


their expression levels and the survival outcomes of LUAD patients via Kaplan-Meier survival analysis (Figure 2). The results showed that patients with high expression levels of $e I F 3 B, e I F 3 D$, or $e I F 3 M$ exhibited worse prognosis than those with low expression levels $\left(p_{\text {eIF3B }}=0.0034\right.$, $p_{\text {eIF3D }}=0.0337$, and $\left.p_{\text {eIF3M }}=0.0094\right)$. On the contrary, the elevated expression of eIF3L predicted better prognosis for our studied patients with LUAD $(p=0.0326)$. No significant differences in prognosis were observed between patients with high and low eIF3A, eIF3C, eIF3E, eIF3H, eIF3I, and eIF3J expression levels.

To verify the robust prognostic roles of eIF3B, eIF3D, $e I F 3 L$, and $e I F 3 M$, the univariate and multivariate Cox regression analyses were performed. Univariate analysis showed that besides the expression levels of the four genes $\left(\mathrm{HR}_{\mathrm{eIF} 3 \mathrm{~B}}=1.563, p=0.004 ; \mathrm{HR}_{\mathrm{eIF} 3 \mathrm{D}}=1.377, p=0.034 ; \mathrm{H}\right.$ $\mathrm{R}_{\mathrm{eIF} 3 \mathrm{~L}}=0.710, p=0.023$; and $\left.\mathrm{HR}_{\mathrm{eIF} 3 \mathrm{M}}=1.521, p=0.006\right)$, pathological stage $(\mathrm{HR}=2.581, p<0.001)$, radiation therapy $(\mathrm{HR}=2.016, p<0.001)$, and residual tumour $(\mathrm{HR}=4.187$, $p<0.001$ ) were also significant prognostic factors (Table 2 ). By running four separate multivariate models (eIF3B, eIF3D, $e I F 3 L$, and $e I F 3 M$ ) adjusted by a series of clinicopathological parameters, it demonstrated that eIF3D expression was an independent risk factor for OS ( $\mathrm{HR}=2.043,95 \% \mathrm{CI}$ : 1.132 $3.689, p=0.018$, Figure $3(\mathrm{~b})$ ), while no significant correlations were observed between other three genes and the OS (Figures 3(a), 3(c), and 3(d)). This result indicated that the overexpression of eIF3D could independently predict poor prognosis for patients with LUAD.

3.4. In Silico Analysis of the Potential Mechanisms Underlying eIF3D Upregulation in LUAD Patients. To explore the mechanisms of eIF3D dysregulation in patients with LUAD, we examined the correlations between eIF3D mRNA expression and its genetic (typically somatic mutations and CNAs) and epigenetic (typically methylation) alterations using the corresponding data in TCGA. Results showed that among 543 cases with somatic mutations measured, only three missense mutations were observed (data not shown); thus, we did not make further statistical analysis between eIF3D expression and its somatic mutations for the insufficient number of mutation cases.

Among 508 cases with DNA CNAs identified, 68 cases (13.4\%) had CNA gains $(+1 /+2), 224$ cases $(44.1 \%)$ had CNA losses (-1/-2), and 216 cases $(42.5 \%)$ were copy neutral (0). Unpaired $t$-test analysis showed both CNA gains and losses had significant influence on eIF3D expression $(p<0.0001$, Figure $4(\mathrm{~b}))$ and that a strong correlation was also observed between eIF3D expression and its linear copy number values by a regression analysis (Pearson $r=0.665$, $p<0.0001$, Figure 4(c)). Besides, Kaplan-Meier survival analysis showed that patients with CNA gains exhibited significantly worse prognosis than those with CNA losses and copy neutral $(p=0.0427$, Figure $4(d))$. As for eIF3D DNA methylation in LUAD, the methylation status of 23 CpG sites was measured by Methylation 450k, and among which nine were hypomethylated in tumour tissues $(n=455)$ compared with the adjacent counterparts $(n=31)$ (Table S1). The regression analysis demonstrated a significantly negative correlation between eIF3D expression and a single CpG site of cg14297023 in tumour tissues (Pearson's $r=-0.2105, p<0.0001$, Figure $4(\mathrm{f})$ ), but no direct correlation was observed between cg14297023 methylation levels and the survival outcomes $(p=0.5788$, Figure 4(g)). Overall, these results indicated that the expression of eIF3D was at least regulated by both CNAs and cg14297023 methylation.

\section{Discussion}

In this study, we examined the mRNA expression profiles of all 13 eIF3 family members and determined their prognostic roles in LUAD patients using corresponding data from TCGA project. The integrated results demonstrated that the overexpression of $e I F 3 D$ could independently predict poor prognosis for patients with LUAD.

Eukaryotic translation initiation depends on ribosomal subunits and, at least, 12 auxiliary proteins named eukaryotic initiation factors (eIFs), and among which eIF3 is the largest and most complex one comprising 13 subunits assembled together in an orderly way. Deregulation of eIF3 expression and/or function has been proposed to play either a causal role or at least contribute to the etiology of various cancer entities. In our study, ten eIF3 subunits were observed aberrantly expressed in LUAD tumour tissues than that of normal counterparts. Studies have revealed that imbalanced expression of single eIF3 subunit may affect the overall expression profiles of the entire eIF3 complex [26], which may explain why the vast majority of eIF3 subunits were observed to be aberrantly expressed in our study. The misregulation of eIF3 subunits may contribute to malignancy via several possible means. First, the altered expression of mammalian eIF3 subunits, especially those in the core regions defined as octamer ( $a, c$, e, f, h, k, l, m) and YLC (Yeast- Like-Core: b, g, i), may impact on the correct assembly of the entire eIF3 complex [26], leading to irregular mRNA translation and causing disease or its quick progression. Second, the overexpression of single eIF3 subunit and/or the resulting upregulation of the entire eIF3 complex would induce a more efficient translation initiation rate of specific mRNAs, which is known as a common feature in cancer [27]. Third, besides translation initiation, the eIF3 subunits may function in other important molecular events or cellular processes associated with a disease phenotype.

The eIF3D, a peripheral subunit attaching to the eIF3 holocomplex via eIF3E, showed very distinct characteristics with its other family members. It is the only subunit whose misregulation affects neither the expression of the other eIF3 subunits nor the integrity of the eIF3 complex but is nonetheless essential for cell proliferation [26]. Instead of the canonical roles of eIF3 in general translation, the essential biological function of eIF3D lies in driving cap-dependent but eIF4E-independent expression of a specific subset of mRNAs encoding proteins with vital cellular roles $[28,29]$. As a noncanonical cap-binding protein, eIF3D was also found to act as an indispensable assistant to help DAP5 which was supposed to initiate only IRES- (internal ribosome entry site-) mediated translation to promote a widespread alternate form of cap-dependent mRNA translation [30]. 


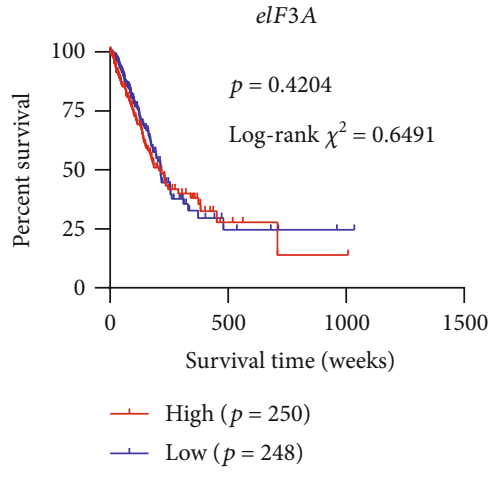

(a)

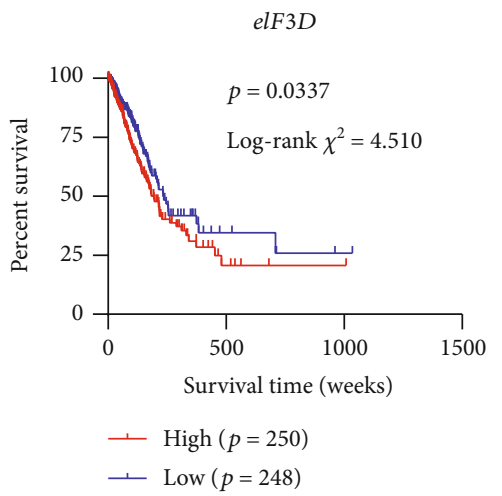

(d)

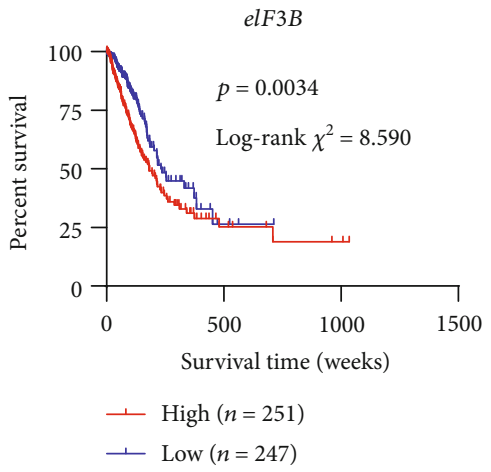

(b)

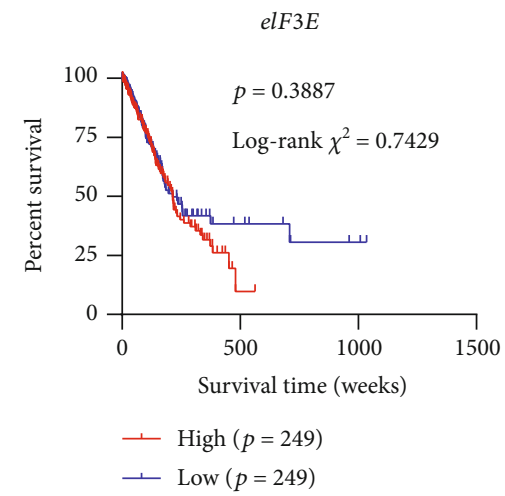

(e)

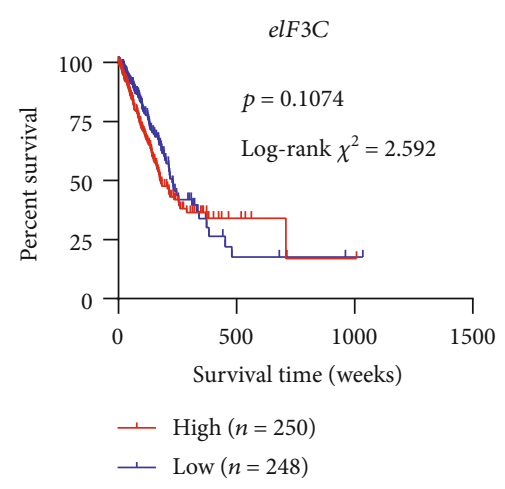

(c)

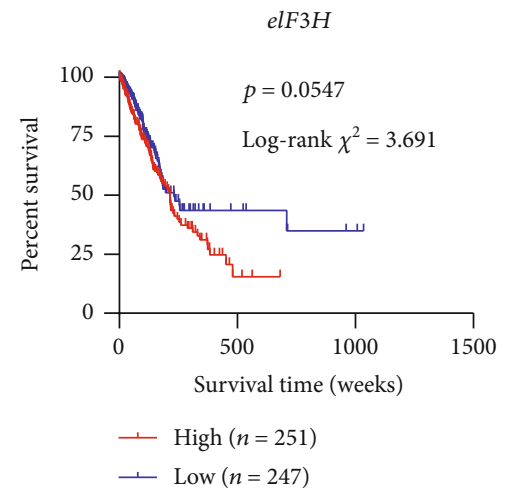

(f)

elF3I
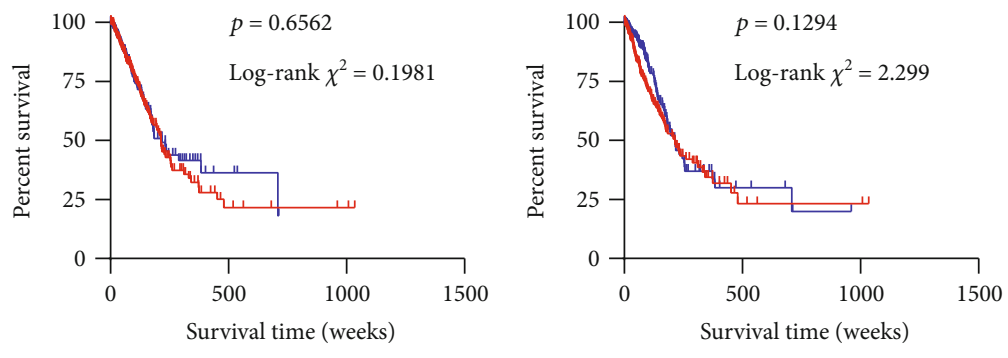

$\longrightarrow$ High $(n=250)$
$\longrightarrow \operatorname{Low}(n=248)$

$\rightarrow$ High $(n=253)$

$\longrightarrow$ Low $(n=245)$

(g)

(h)

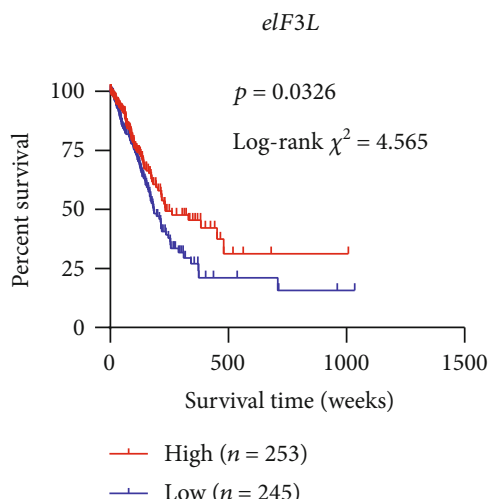

(i)

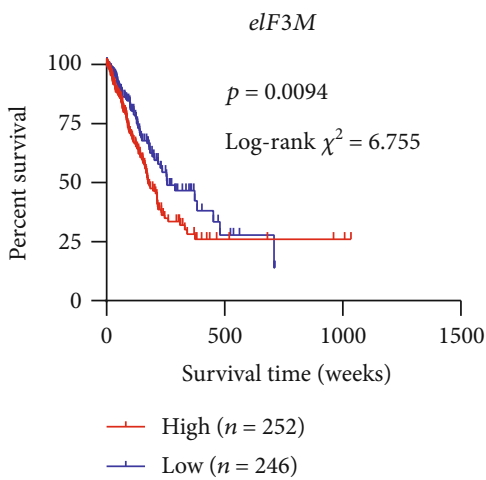

(j)

Figure 2: Kaplan-Meier survival analysis of differentially expressed subunits of $e I F 3$ for OS in LUAD patients. $\chi^{2}$ and $p$ values assessed by log-rank test and number of patients in each group are provided in the figure. 
TABLE 2: Univariate analysis of OS in LUAD patients.

\begin{tabular}{|c|c|c|c|}
\hline Parameters & \multicolumn{3}{|c|}{ Univariate analysis } \\
\hline \multicolumn{4}{|l|}{ Age } \\
\hline$<65(N=220)$ & 1.000 & & \\
\hline$\geq 65(N=273)$ & 1.145 & $0.849-1.545$ & 0.375 \\
\hline \multicolumn{4}{|l|}{ Gender } \\
\hline Female $(N=273)$ & 1.000 & & \\
\hline Male $(N=230)$ & 1.030 & $0.768-1.382$ & 0.843 \\
\hline \multicolumn{4}{|l|}{ Pathological stage } \\
\hline $\mathrm{I} / \mathrm{II}(N=392)$ & 1.000 & & \\
\hline III/IV $(N=103)$ & 2.581 & $1.884-3.535$ & $<0.001^{*}$ \\
\hline \multicolumn{4}{|l|}{ Radiation therapy } \\
\hline No $(N=392)$ & 1.000 & & \\
\hline Yes $(N=58)$ & 2.016 & $1.372-2.962$ & $<0.001^{*}$ \\
\hline \multicolumn{4}{|l|}{ Residual tumour } \\
\hline $\mathrm{R} 0(N=336)$ & 1.000 & & \\
\hline $\mathrm{R} 1 / \mathrm{R} 2(N=16)$ & 4.187 & $2.333-7.512$ & $<0.001^{*}$ \\
\hline \multicolumn{4}{|l|}{ Smoking history } \\
\hline $1(N=72)$ & 1.000 & & \\
\hline $2 / 3 / 4 / 5(N=417)$ & 0.888 & $0.588-1.341$ & 0.571 \\
\hline \multicolumn{4}{|l|}{ Mutations } \\
\hline No $(N=125)$ & 1.000 & & \\
\hline Yes $(N=91)$ & 0.866 & $0.558-1.346$ & 0.523 \\
\hline \multicolumn{4}{|l|}{ Targeted therapy } \\
\hline No $(N=299)$ & 1.000 & & \\
\hline Yes $(N=149)$ & 1.159 & $0.830-1.620$ & 0.386 \\
\hline \multicolumn{4}{|l|}{ EIF3B expression } \\
\hline Low $(N=247)$ & 1.000 & & \\
\hline $\operatorname{High}(N=251)$ & 1.563 & $1.156-2.111$ & $0.004^{*}$ \\
\hline \multicolumn{4}{|l|}{ eIF3D expression } \\
\hline Low $(N=248)$ & 1.000 & & \\
\hline $\operatorname{High}(N=250)$ & 1.377 & $1.024-1.854$ & $0.034^{*}$ \\
\hline \multicolumn{4}{|l|}{ EIF3L expression } \\
\hline Low $(N=245)$ & 1.000 & & \\
\hline High $(N=253)$ & 0.710 & $0.528-0.955$ & $0.023^{*}$ \\
\hline \multicolumn{4}{|l|}{ EIF3M expression } \\
\hline Low $(N=246)$ & 1.000 & & \\
\hline High $(N=252)$ & 1.521 & $1.127-2.053$ & $0.006^{*}$ \\
\hline
\end{tabular}

${ }^{*}$ Indicates statistical significance $(p<0.05)$.

The overexpression of eIF3D has been observed to promote cell proliferation, migration, or/and tumour growth in several cancer entities, including ovarian cancer [31], renal cell carcinoma [32], gastric cancer (GC) [33], and gallbladder cancer (GBC) [34]. As being discovered in our study, all these high levels of eIF3D are associated with advanced tumour stage, indicating its potential role in tumour development. The eIF3D knockdown researches conducted in a series of cancer cell lines involving breast cancer [35], NSCLC [36], melanoma [37], acute myeloid leukaemia [38], and colon cancer [39] revealed that the deletion of eIF3D caused a significant reduction in cell proliferation and colony formation due to an arrest of cell cycle at G2/M phase, suggesting its key role in cell cycle control and apoptosis. Previous studies demonstrated that eIF3D affected cancer cell growth via multiple signaling pathways. Zhang et al. proposed that eIF3D exerted the tumour-promoting activities through GRK2-mediated activation of PI3K/AKT pathway in GBC [34]. Fan et al. found that knockdown of eIF3D inhibited the activation of $\mathrm{Wnt} / \beta$-catenin signaling pathway in breast cancer cell lines via blockade of the expression of $\beta$-catenin, cyclin D1, and c-Myc [35]. By exploring the modifications of effector proteins in signaling pathways responsible for cell growth and apoptosis, the activations of three cancer-related molecules-AKT, HSP27, and SAPK/JNK-were found to be reduced by eIF3D knockdown in NSCLC cells [36]. Moreover, a series of phosphorylation upregulation was observed along with downregulation of $e I F 3 D$ in colon cancer cells, including AMPK $\alpha$, Bad, PRAS409, SAPK/JNK, and GSK3 $\beta$, as well as the cleavage of PARP [39]. These mechanisms would help to explain the oncogenic properties of eIF3D in patients with LUAD. In our study, the prognostic value of eIF3D was confirmed in LUAD independent of multiple clinicopathological parameters. Being consistent with our findings, the strong correlations between high eIF3D expression and poor OS outcomes were also observed in patients with GC [33] and GBC [34], supporting its high potential role to serve as a useful prognostic marker and therapeutic target for the treatment of these cancers.

In this study, by assessing the potential mechanisms underlying eIF3D upregulation in LUAD, we found that the eIF3D dysregulation was partly regulated by the CNAs, as well as the hypomethylation of $\operatorname{cg} 14297023$ site which locates in the $3^{\prime}$ UTR region of eIF3D gene according to the MethHC database (http://methhc.mbc.nctu.edu.tw/php/index.php) [40]. It is clear that a CNA is generally positively associated with the expression level of its corresponding gene. However, as for the role of DNA methylation in the $3^{\prime}$ UTR on the influence of gene expression, far less is known than a comprehensive understanding of that in the gene promoter region. Recently, McGuire et al. proposed an explanation that DNA methylation in $3^{\prime}$ UTR might control gene expression via two potential ways [41]. First, it may influence gene expression by increasing binding of proteins with methylation-binding domains or inhibiting other special protein binding or mask sequence recognition $[42,43]$. Another potential explanation is that if different lengths of the $3^{\prime}$ UTR are dependent on methylation, transcripts with shorter $3^{\prime}$ UTRs would have greater mRNA stability and thereby higher gene expression [44]. On the contrary, studies also found that gene body methylation would reflect as a consequence of higher gene expression, rather than as a cause [45]. Therefore, the specific role of hypomethylation of cg14297023 site on eIF3D expression in LUAD cannot be concluded yet based on these hypotheses. Beyond that, we could not exclude other genetic or epigenetic mechanisms influencing the transcription and translation of eIF3D. Thus, 


Parameters
Age
Gender
Pathological stage
Radiation therapy
Residual tumor
Smoking history
Mutation in KRAS/EGFR/ALK
Targeted molecular therapy
eIF3B expression

$\begin{array}{ccr}\text { Hazard ratio (HR) } & 95 \% \text { CI of HR } & p \text { value } \\ 1.236 & 0.683-2.237 & 0.483 \\ 1.026 & 0.569-1.852 & 0.931 \\ 2.102 & 1.126-3.924 & 0.020 \\ 2.225 & 1.038-4.773 & 0.040 \\ 3.591 & 1.388-9.292 & 0.008 \\ 0.755 & 0.340-1.677 & 0.491 \\ 1.205 & 0.685-2.119 & 0.518 \\ 0.578 & 0.264-1.266 & 0.170 \\ 1.576 & 0.912-2.724 & 0.103\end{array}$

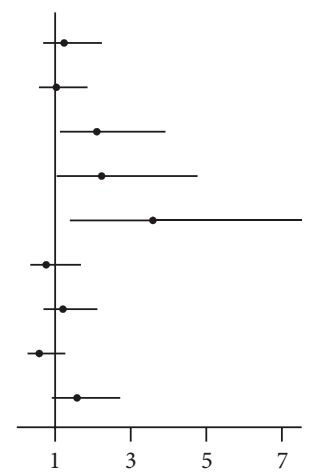

Parameters

Age

Gender

Pathological stage

Radiation therapy

Residual tumor

Smoking history

Mutation in KRAS/EGFR/ALK

Targeted molecular therapy

eIF3D expression

Hazard ratio (HR)

(a)

$\begin{array}{lll}1.149 & 0.636-2.077 & 0.646 \\ 0.993 & 0.554-1.782 & 0.981 \\ 1.774 & 0.946-3.327 & 0.074 \\ 1.864 & 0.869-4.000 & 0.110 \\ 3.456 & 1.329-8.991 & 0.011 \\ 0.796 & 0.364-1.738 & 0.566 \\ 1.314 & 0.743-2.322 & 0.347 \\ 0.696 & 0.322-1.502 & 0.355 \\ 2.043 & 1.132-3.689 & 0.018\end{array}$

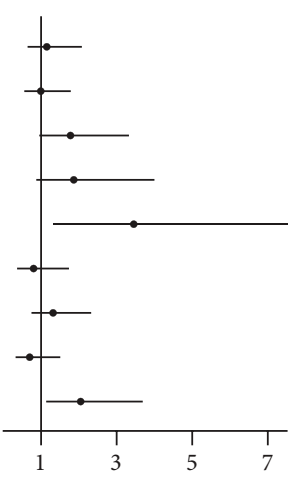

(b)

Parameters
Age
Gender
Pathological stage
Radiation therapy
Residual tumor
Smoking history
Mutation in KRAS/EGFR/ALK
Targeted molecular therapy
eIF3L expression

Hazard ratio (HR)

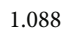

95\% CI of $\mathrm{HR}$ 0.588-2.011

$p$ value

1.088
0.936

0.520-1.682

1.113-3.791

0.973-4.584

1.328-8.939

0.305-1.6090

0.682-2.080

0.286-1.331

0.664-2.277

0.789
0.824
0.021
0.059
0.011
0.401
0.540
0.218
0.512

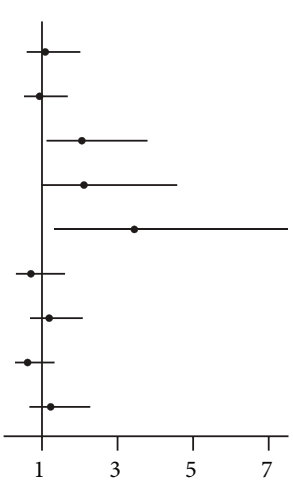

(c)

Parameters

Age

Gender

Pathological stage

Radiation therapy

Residual tumor

Smoking history

Mutation in KRAS/EGFR/ALK

Targeted molecular therapy

eIF3 $M$ expression
Hazard ratio (HR)

1.141

1.018

2.103

2.082

3.417

0.765

1.193

0.595

1.370
95\% CI of HR

0.636-2.047

0.556-1.864

1.124-3.937

$0.972-4.458$

1.322-8.829

0.342-1.710

0.684-2.081

0.272-1.299

$0.778-2.414$

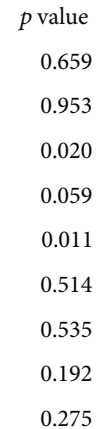

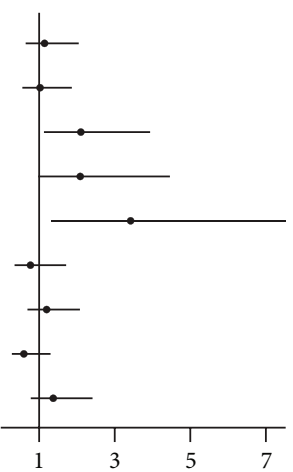

(d)

Figure 3: Forest plots for multivariate Cox regression models of four eIF3 subunits: (a) eIF3B; (b) eIF3D; (c) eIF3L; (d) eIF3M. 


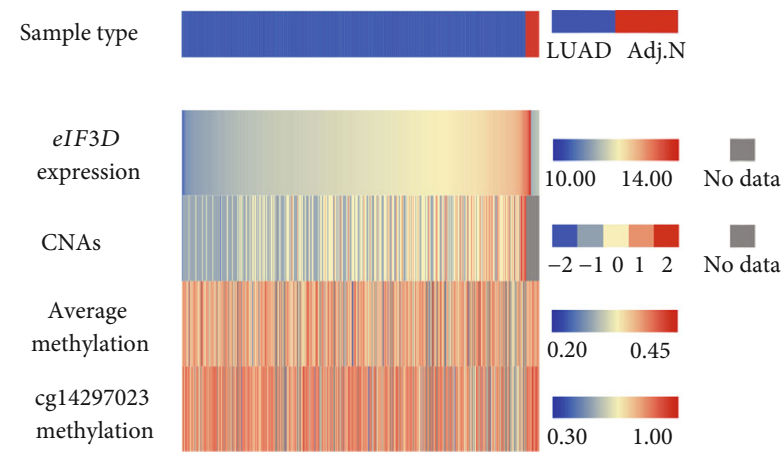

(a)

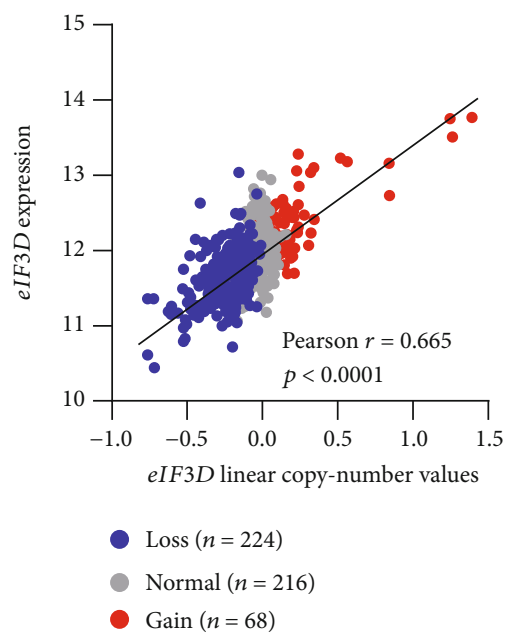

(c)

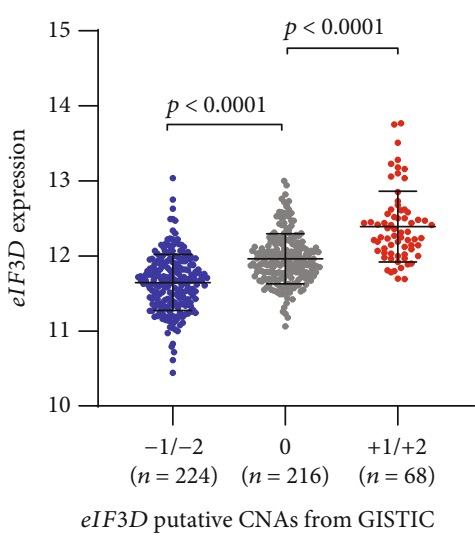

(b)

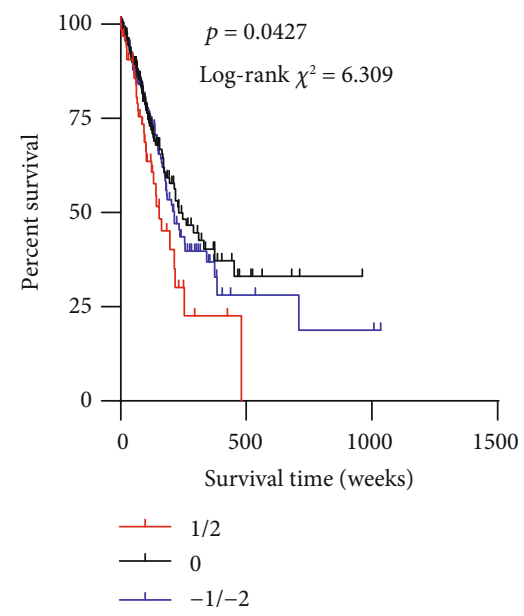

(d)

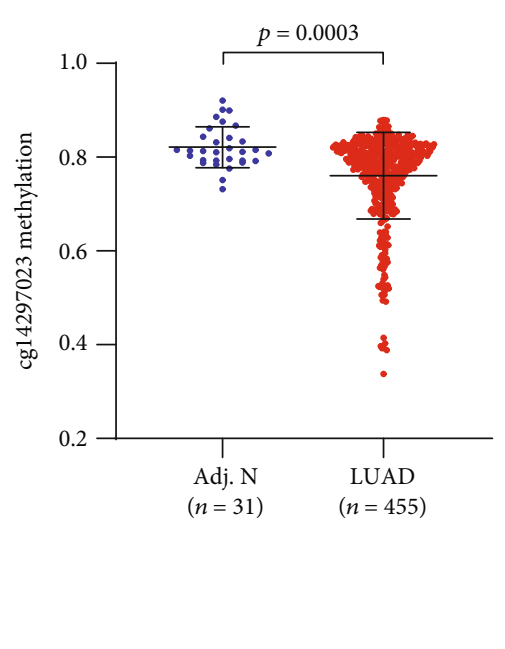

(e)

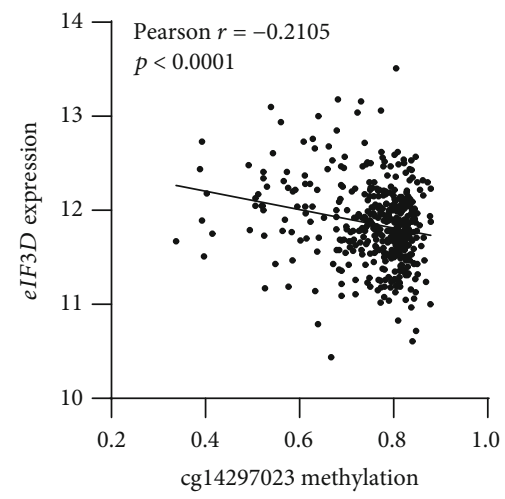

(f)

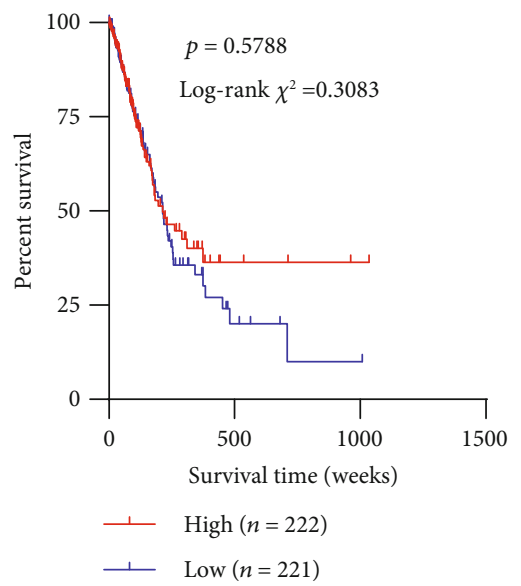

(g)

FIGURE 4: Copy number alterations and DNA methylation analysis of eIF3D in LUAD patients. (a) Heatmap showing eIF3D expression and corresponding DNA copy number alterations, averaged DNA methylation, and methylation in cg14297023. (b) Plot chart showing eIF3D expression in different CNA groups. (c) Linear regression analysis of the correlation between eIF3D expression and its linear copy number values. (d) Kaplan-Meier survival analysis of different eIF3D CNA levels for OS in patients with LUAD. (e) Plot chart showing DNA methylation of cg14297023 site in LUAD tissues and adjacent normal tissues. (f) Linear regression analysis of the correlation between cg14297023 methylation and eIF3D expression. (g) Kaplan-Meier survival analysis of different methylation levels in cg14297023 for OS in patients with LUAD. Adj. N: adjacent normal tissues; GISTIC: Genomic Identification of Significant Targets in Cancer [25]. 
experimental studies are required to further explore the role and other potential mechanisms of eIF3D in LUAD.

With regard to other subunits of eIF3, their misregulation and potential roles in tumour progression or survival outcome have been observed in a vast range of cancer entities (reviewed by $[18,46,47]$ ). In our study, even though their independent prognostic roles were not observed, we still got some noteworthy results. A previous study by Cattie et al. has revealed that loss-of-function mutations in eIF3L gene resulted in a $40 \%$ extension in lifespan in Caenorhabditis elegans, indicating its pivotal functional role in the regulation of cellular and organismal responses to aging [48]. By examining the links between gene expression levels and clinical features, the highly expressed eIF3L was found positively associated with LUAD patients with ages no lower than 65 (151/122 vs. $93 / 126, p=0.0050$, Table 1$)$ in our study, adding to the evidence of its regulatory role during aging, as Cattie et al. proposed [48]. As an associated factor locating at the more peripheral position of the eIF3 complex, eIF3J is known to be weakly and unstably interacted with the complex and most likely plays only a supportive role in the overall translational efficiency in human cells [49]. However, little is known in the relation of eIF3J and carcinogenesis by now $[15,50$, 51]. The mutations in KRAS/EGFR/ALK are the most common "driver" mutations detected in LUAD and are considered to play pivotal roles in carcinogenesis at multiple levels, thus acting as important genomic-guided therapeutic targets in LUAD (reviewed by [3]). In our study, the discovery of different expression levels of eIF3J occurring in patients with or without mutations in KRAS/EGFR/ALK ( $86 / 46$ vs. $47 / 48, p=0.0206$, Table 1 ) may provide a clue of its potential impact on LUAD carcinogenesis accompanied by KRAS/EGFR/ALK genomic alterations. Besides, nine eIF3 subunits beyond eIF3D were found aberrantly expressed in LUAD tissues and in which the expression levels of five subunits $(e I F 3 B, e I F 3 C, e I F 3 E$, eIF3H, and $e I F 3 J)$ increased in LUAD patients with high tumour stage, implicating their roles in the maintenance or progression of LUAD. Therefore, it is meaningful to further explore the potential roles and underlying molecular mechanisms of action of these eIF3 subunits as well as $e I F 3 D$ in LUAD in the future.

\section{Conclusions}

High eIF3D expression might serve as a valuable independent prognostic indicator of shorter overall survival in patients with LUAD.

\section{Data Availability}

The data used to support the findings of this study are available from the corresponding author upon request.

\section{Conflicts of Interest}

All authors declare that they have no conflicts of interest.

\section{Acknowledgments}

This work was supported by a National Natural Science Foundation cultivation project of Jining Medical University, China (JY2017FY012), and an open project of Shanxi Provincial Key Laboratory of Forensic Science, China (SFM2019002).

\section{Supplementary Materials}

Table S1: methylation status of eIF3D CpG sites in LUAD tissues and adjacent normal tissues. (Supplementary Materials)

\section{References}

[1] W. D. Travis, L. B. Travis, and S. S. Devesa, "Lung cancer," Cancer, vol. 75, no. S1, pp. 191-202, 1995.

[2] T. V. Denisenko, I. N. Budkevich, and B. Zhivotovsky, "Cell death-based treatment of lung adenocarcinoma," Cell Death \& Disease, vol. 9, no. 2, pp. 117-130, 2018.

[3] R. Chalela, V. Curull, C. Enríquez, L. Pijuan, B. Bellosillo, and J. Gea, "Lung adenocarcinoma: from molecular basis to genome-guided therapy and immunotherapy," Journal of Thoracic Disease, vol. 9, no. 7, pp. 2142-2158, 2017.

[4] F. Tian, C. T. Yu, W. D. Ye, and Q. Wang, "Cinnamaldehyde induces cell apoptosis mediated by a novel circular RNA hsa_circ_0043256 in non-small cell lung cancer," Biochemical and Biophysical Research Communications, vol. 493, no. 3, pp. 1260-1266, 2017.

[5] D. Silvera, R. Arju, F. Darvishian et al., "Essential role for eIF4GI overexpression in the pathogenesis of inflammatory breast cancer," Nature Cell Biology, vol. 11, no. 7, pp. 903908, 2009.

[6] F. Meric and K. K. Hunt, "Translation initiation in cancer: a novel target for therapy," Molecular Cancer Therapeutics, vol. 1, no. 11, pp. 971-979, 2002.

[7] R. Spilka, C. Ernst, H. Bergler et al., "eIF3a is over-expressed in urinary bladder cancer and influences its phenotype independent of translation initiation," Cellular Oncology, vol. 37, no. 4, pp. 253-267, 2014.

[8] G. Xu, H. Yu, X. Shi et al., "Cisplatin sensitivity is enhanced in non-small cell lung cancer cells by regulating epithelialmesenchymal transition through inhibition of eukaryotic translation initiation factor 5A2," BMC Pulmonary Medicine, vol. 14, article 174, 2014.

[9] C. Demosthenous, J. J. Han, M. J. Stenson et al., “Translation initiation complex eIF4F is a therapeutic target for dual mTOR kinase inhibitors in non-Hodgkin lymphoma," Oncotarget, vol. 6, no. 11, pp. 9488-9501, 2015.

[10] A. Miluzio, S. Oliveto, E. Pesce et al., "Expression and activity of eIF6 trigger malignant pleural mesothelioma growth in vivo," Oncotarget, vol. 6, no. 35, pp. 37471-37485, 2015.

[11] C. Vaysse, C. Philippe, Y. Martineau et al., "Key contribution of eIF4H-mediated translational control in tumor promotion," Oncotarget, vol. 6, no. 37, pp. 39924-39940, 2015.

[12] L. Dai, Z. Lin, Y. Cao, Y. Chen, Z. Xu, and Z. Qin, "Targeting EIF4F complex in non-small cell lung cancer cells," Oncotarget, vol. 8, no. 33, pp. 55731-55735, 2017.

[13] N. Golob-Schwarzl, S. Krassnig, A. M. Toeglhofer et al., "New liver cancer biomarkers: PI3K/AKT/mTOR pathway members 
and eukaryotic translation initiation factors," European Journal of Cancer, vol. 83, pp. 56-70, 2017.

[14] N. Golob-Schwarzl, C. Schweiger, C. Koller et al., "Separation of low and high grade colon and rectum carcinoma by eukaryotic translation initiation factors 1, 5 and 6," Oncotarget, vol. 8, no. 60, pp. 101224-101243, 2017.

[15] A. Unbehaun, S. I. Borukhov, C. U. Hellen, and T. V. Pestova, "Release of initiation factors from $48 \mathrm{~S}$ complexes during ribosomal subunit joining and the link between establishment of codon-anticodon base-pairing and hydrolysis of eIF2-bound GTP," Genes \& Development, vol. 18, no. 24, pp. 3078-3093, 2004.

[16] Z. Dong and J. T. Zhang, "Initiation factor eIF3 and regulation of mRNA translation, cell growth, and cancer," Critical Reviews in Oncology/Hematology, vol. 59, no. 3, pp. 169-180, 2006.

[17] J. Y. Yin, Z. Dong, Z. Q. Liu, and J. T. Zhang, "Translational control gone awry: a new mechanism of tumorigenesis and novel targets of cancer treatments," Bioscience Reports, vol. 31, no. 1, pp. 1-15, 2011.

[18] R. Spilka, C. Ernst, A. K. Mehta, and J. Haybaeck, "Eukaryotic translation initiation factors in cancer development and progression," Cancer Letters, vol. 340, no. 1, pp. 9-21, 2013.

[19] R. Pincheira, Q. Chen, and J. T. Zhang, "Identification of a $170-\mathrm{kDa}$ protein over-expressed in lung cancers," British Journal of Cancer, vol. 84, no. 11, pp. 1520-1527, 2001.

[20] S. Matsuda, R. Katsumata, T. Okuda et al., "Molecular cloning and characterization of human MAWD, a novel protein containing WD-40 repeats frequently overexpressed in breast cancer," Cancer Research, vol. 60, no. 1, pp. 13-17, 2000.

[21] M. Grzmil, T. Rzymski, M. Milani et al., "An oncogenic role of eIF3e/INT6 in human breast cancer," Oncogene, vol. 29, no. 28, pp. 4080-4089, 2010.

[22] Y. N. Zhu, Expression of eIF3a in gastric cancer, Central South University, 2012.

[23] A. Marchetti, F. Buttitta, S. Pellegrini, G. Bertacca, and R. Callahan, "Reduced expression of INT-6/eIF3-p 48 in human tumors," International Journal of Oncology, vol. 18, no. 1, pp. 175-179, 2001.

[24] J. S. Huang, C. C. Chao, T. L. Su et al., "Diverse cellular transformation capability of overexpressed genes in human hepatocellular carcinoma," Biochemical and Biophysical Research Communications, vol. 315, no. 4, pp. 950-958, 2004.

[25] C. H. Mermel, S. E. Schumacher, B. Hill, M. L. Meyerson, R. Beroukhim, and G. Getz, "GISTIC2.0 facilitates sensitive and confident localization of the targets of focal somatic copy-number alteration in human cancers," Genome biology, vol. 12, no. 4, p. R41, 2011.

[26] S. Wagner, A. Herrmannová, D. Šikrová, and L. S. Valášek, "Human eIF3b and eIF3a serve as the nucleation core for the assembly of eIF3 into two interconnected modules: the yeastlike core and the octamer," Nucleic Acids Research, vol. 44, no. 22, pp. 10772-10788, 2016.

[27] M. Bhat, N. Robichaud, L. Hulea, N. Sonenberg, J. Pelletier, and I. Topisirovic, "Targeting the translation machinery in cancer," Nature Reviews Drug Discovery, vol. 14, no. 4, pp. 261-278, 2015.

[28] A. S. Lee and P. J. Kranzusch, "eIF3 targets cell-proliferation messenger RNAs for translational activation or repression," Nature, vol. 522, no. 7554, pp. 111-114, 2015.

[29] A. S. Lee, P. J. Kranzusch, J. A. Doudna, and J. H. Cate, "eIF3d is an mRNA cap-binding protein that is required for special- ized translation initiation," Nature, vol. 536, no. 7614, pp. 96-99, 2016.

[30] C. de la Parra, A. Ernlund, A. Alard, K. Ruggles, B. Ueberheide, and R. J. Schneider, "A widespread alternate form of capdependent mRNA translation initiation," Nature Communications, vol. 9, no. 1, p. 3068, 2018.

[31] Y. Lin, R. Zhang, and P. Zhang, "Eukaryotic translation initiation factor 3 subunit D overexpression is associated with the occurrence and development of ovarian cancer," FEBS Open Bio, vol. 6, no. 12, pp. 1201-1210, 2016.

[32] X. W. Pan, L. Chen, Y. Hong et al., "EIF3D silencing suppresses renal cell carcinoma tumorigenesis via inducing G2/M arrest through downregulation of Cyclin B1/CDK1 signaling," International Journal of Oncology, vol. 48, no. 6, pp. 2580-2590, 2016.

[33] J. He, X. Wang, J. Cai, W. Wang, and X. Qin, “High expression of eIF3d is associated with poor prognosis in patients with gastric cancer," Cancer Management and Research, vol. 9, pp. 539-544, 2017.

[34] F. Zhang, S. Xiang, Y. Cao et al., "EIF3D promotes gallbladder cancer development by stabilizing GRK2 kinase and activating PI3K-AKT signaling pathway," Cell Death \& Disease, vol. 8, no. 6, article e2868, 2017.

[35] Y. Fan and Y. Guo, "Knockdown of eIF3D inhibits breast cancer cell proliferation and invasion through suppressing the Wnt/ $\beta$-catenin signaling pathway," International Journal of Clinical and Experimental Pathology, vol. 8, no. 9, pp. 10420-10427, 2015.

[36] Z. Lin, L. Xiong, and Q. Lin, "Knockdown of eIF3d inhibits cell proliferation through $\mathrm{G} 2 / \mathrm{M}$ phase arrest in non-small cell lung cancer," Medical Oncology, vol. 32, no. 7, pp. 183-190, 2015.

[37] H. Li, F. Zhou, H. Wang et al., "Knockdown of EIF3D suppresses proliferation of human melanoma cells through G2/M phase arrest," Biotechnology and Applied Biochemistry, vol. 62, no. 5, pp. 615-620, 2015.

[38] G. Z. Liu, J. Z. Liu, X. Q. Li et al., "Knockdown of eukaryotic translation initiation factor 3 subunit D (eIF3D) inhibits proliferation of acute myeloid leukemia cells," Molecular and Cellular Biochemistry, vol. 438, no. 1-2, pp. 191-198, 2018.

[39] X. Yu, B. Zheng, and R. Chai, "Lentivirus-mediated knockdown of eukaryotic translation initiation factor 3 subunit D inhibits proliferation of HCT116 colon cancer cells," Bioscience Reports, vol. 34, no. 6, article e00161, 2014.

[40] W. Y. Huang, S. D. Hsu, H. Y. Huang et al., "MethHC: a database of DNA methylation and gene expression in human cancer," Nucleic Acids Research, vol. 43, no. D1, pp. D856D861, 2015.

[41] M. H. McGuire, S. M. Herbrich, S. K. Dasari et al., "Pan-cancer genomic analysis links $3^{\prime}$ UTR DNA methylation with increased gene expression in T cells," eBioMedicine, vol. 43, pp. 127-137, 2019.

[42] O. Bogdanović and G. J. Veenstra, "DNA methylation and methyl-CpG binding proteins: developmental requirements and function," Chromosoma, vol. 118, no. 5, pp. 127-137, 2009.

[43] X. Zou, W. Ma, I. A. Solov'yov, C. Chipot, and K. Schulten, "Recognition of methylated DNA through methyl-CpG binding domain proteins," Nucleic Acids Research, vol. 40, no. 6, pp. 2747-2758, 2012. 
[44] C. Mayr and D. P. Bartel, "Widespread shortening of 3'UTRs by alternative cleavage and polyadenylation activates oncogenes in cancer cells," Cell, vol. 138, no. 4, pp. 673-684, 2009.

[45] J. Du, L. M. Johnson, S. E. Jacobsen, and D. J. Patel, "DNA methylation pathways and their crosstalk with histone methylation," Nature Reviews. Molecular Cell Biology, vol. 16, no. 9, pp. 519-532, 2015.

[46] Y. Yin, J. Long, Y. Sun et al., "The function and clinical significance of eIF3 in cancer," Gene, vol. 5, no. 673, pp. 130-133, 2018.

[47] A. Gomes-Duarte, R. Lacerda, J. Menezes, and L. Romão, "eIF3: a factor for human health and disease," RNA Biology, vol. 15, no. 1, pp. 26-34, 2018.

[48] D. J. Cattie, C. E. Richardson, K. C. Reddy et al., "Mutations in nonessential eIF3k and eIF3l genes confer lifespan extension and enhanced resistance to ER stress in Caenorhabditis elegans," PLoS Genetics, vol. 12, no. 9, article e1006326, 2016.

[49] S. Wagner, A. Herrmannová, R. Malík, L. Peclinovská, and L. S. Valášek, "Functional and biochemical characterization of human eukaryotic translation initiation factor 3 in living cells," Molecular and Cellular Biology, vol. 34, no. 16, pp. 3041-3052, 2014.

[50] H. B. Fraser, A. E. Hirsh, G. Giaever, J. Kumm, and M. B. Eisen, "Noise minimization in eukaryotic gene expression," PLoS Biology, vol. 2, no. 6, article e137, 2004.

[51] C. S. Fraser, "The molecular basis of translational control," Progress in Molecular Biology and Translational Science, vol. 90, pp. 1-51, 2009. 


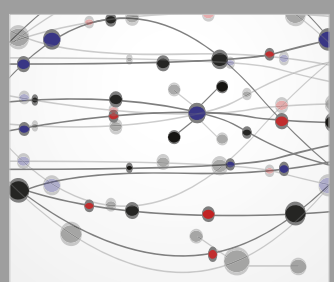

The Scientific World Journal
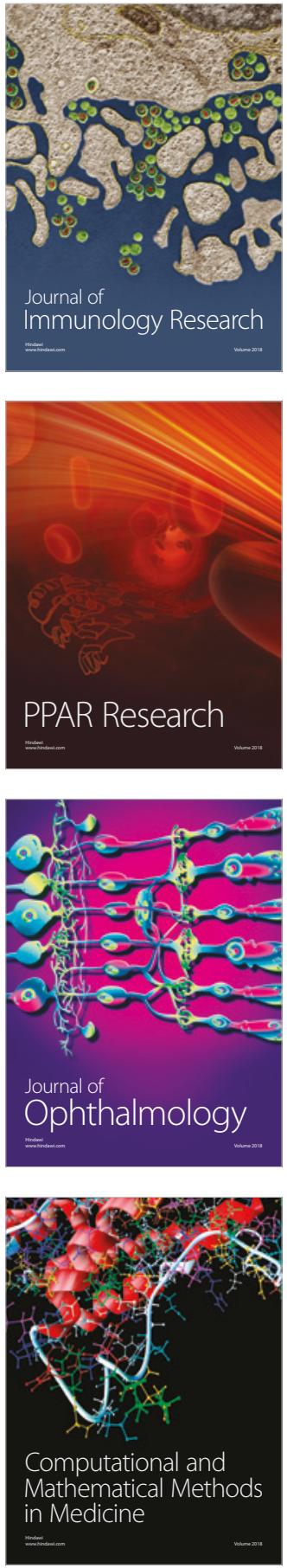

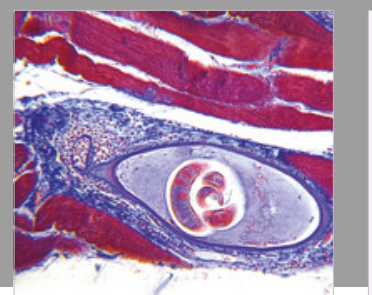

Gastroenterology Research and Practice

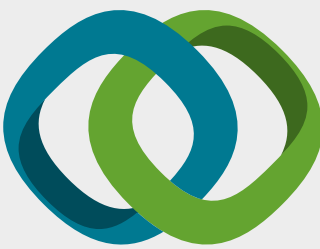

\section{Hindawi}

Submit your manuscripts at

www.hindawi.com
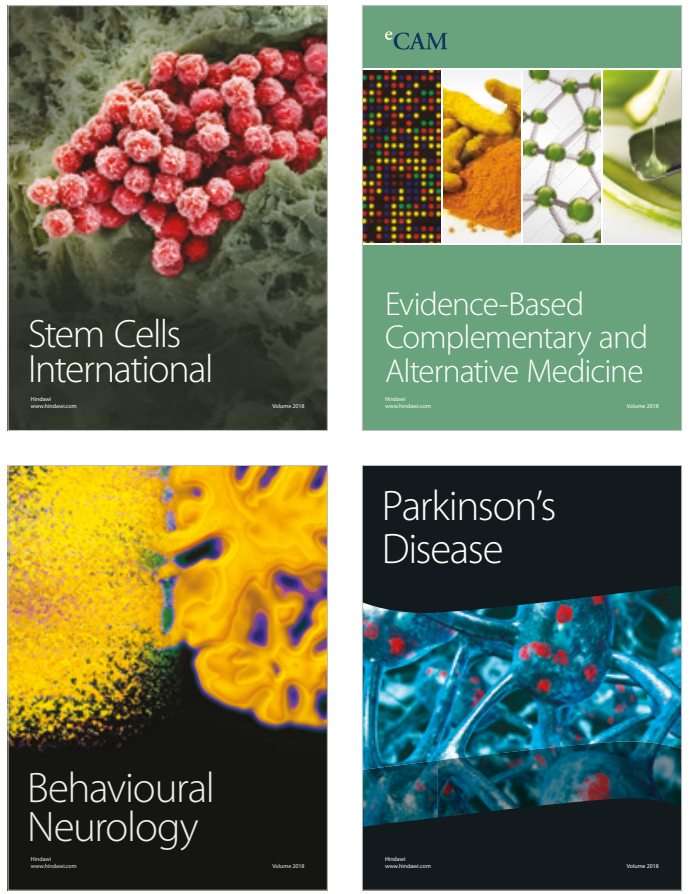

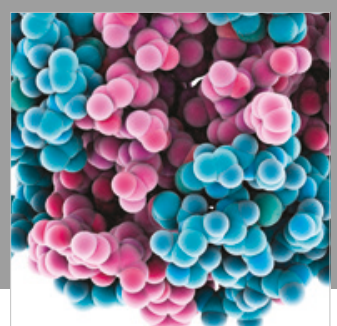

ournal of

Diabetes Research

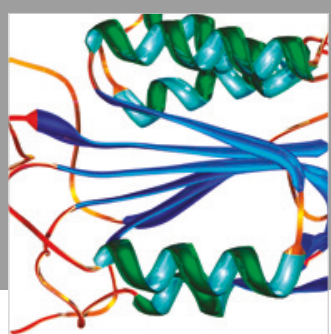

Disease Markers
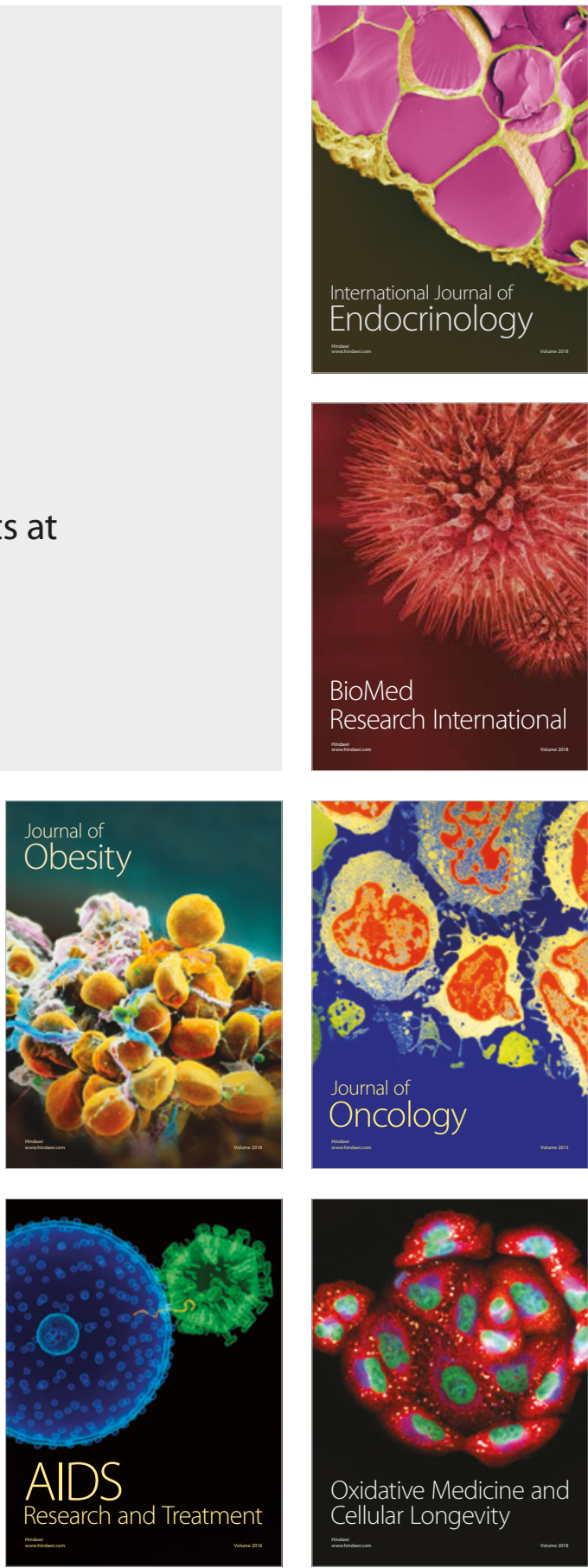